J. Appl. Numer. Optim. 1 (2019), No. 3, pp. 253-265

Available online at http://jano.biemdas.com

https://doi.org/10.23952/jano.1.2019.3.05

\title{
EKELAND VARIATIONAL PRINCIPLES FOR VECTOR EQUILIBRIUM PROBLEMS WITH SOLID ORDERING CONES
}

\author{
CÉSAR GUTIÉRREZ \\ IMUVA (Institute of Mathematics of University of Valladolid), \\ Edificio LUCIA, Campus Miguel Delibes, Paseo de Belén S/N, 47011, Valladolid, Spain
}

\begin{abstract}
This paper concerns with Ekeland variational principles for vector bifunctions. It is assumed that the topological interior of the ordering cone in the final space of the bifunction is nonempty. The main results are stated by nonlinear scalarization through the well-known Gerstewitz functional, and involve a new lower-semicontinuity concept for vector functions and a generalization of the so-called triangle inequality property of a vector bifunction. Some recent Ekeland variational principles of the literature derived for a kind of Henig approximate solutions of vector equilibrium problems are improved as they are obtained by weaker assumptions.
\end{abstract}

Keywords. Vector equilibrium problem; Ekeland variational principle; Scalarization; Approximate efficient solution.

2010 Mathematics Subject Classification. 58E30, 90C33, 49J40, 90C29, 90C48.

\section{INTRODUCTION}

The so-called Ekeland variational principle $[8,9]$ is one of the most important mathematical tools in Nonlinear Analysis and Optimization. That is why it has been extended to different frameworks.

The paper deals with versions of this result for vector bifunctions. Nowadays, these variational principles are a growing research line (see $[1,4,6,10,14,15,19,20,21])$ since they can be formulated as vector equilibrium problems, which encompass several really important problems, like vector optimization problems, vector variational inequalities and vector complementary problems (see $[2,3,7,11,17]$ and the references therein).

This work is motivated by the recent Ekeland variational principles in [15], where they are derived by considering the so-called Gerstewitz scalarization functional and a kind of proper approximate solutions of vector equilibrium problems. These solutions are just weak approximate solutions with respect to certain dilating cones. Therefore, the corresponding Ekeland variational principles can be analyzed in the general framework of a vector equilibrium problem whose ordering cone has nonempty topological interior. As long as we know, this approach was introduced twenty-seven years ago by Tammer [23].

In this setting, more general Ekeland variational principles will be obtained as a result of applying the same scalarization approach to sharper concepts. On the one hand, the notion of cone properness due to Hernández and Rodríguez-Marín [16] and a new lower semicontiunity

E-mail address: cesargv@ mat.uva.es.

Received June 23, 2019; Accepted October 16, 2019.

(C)2019 Journal of Applied and Numerical Optimization 
notion for vector functions (see Definition 3.3), which is the vector counterpart of the so-called strict-decreasingly lower-semicontinuity [5]. On the other hand, a new triangle inequality property (see Definition 3.6) for vector bifunctions. Notice that the last two notions are introduced in this paper for the first time and they would be interesting in themselves.

The paper is structured as follows. In Section 2, the framework and the main notations are fixed. In addition, some basic mathematical tools, as the so-called Gerstewitz functional and its properties, are recalled. In Section 3, the notion of strict $q$-decreasingly $D$-lowersemicontinuous vector function and the triangle inequality property in $-D$ by $q$ at a point for a vector bifunction are introduced and characterized by scalarization. After that, several Ekeland variational principles for vector equilibrium problems are stated. Finally, it is shown that the obtained results improve some corresponding ones of the literature since they are derived by weaker assumptions.

\section{PRELIMINARIES}

From now on, $(X, d)$ denotes a complete metric space and $Y$ stands for a real topological linear space. Moreover, $Y$ is assumed to be ordered by a convex cone $D \subset Y$ via the binary relation $\leq_{D}$ :

$$
y_{1}, y_{2} \in Y, \quad y_{1} \leq_{D} y_{2} \Longleftrightarrow y_{2}-y_{1} \in D .
$$

For each $y_{1}, y_{2} \in Y$, the notation $y_{1}<_{D} y_{2}$ means $y_{1} \leq_{D} y_{2}$ and $y_{1} \neq y_{2}$ (i.e., $y_{2}-y_{1} \in D \backslash\{0\}$ ).

We denote by $\operatorname{int} M$ and bd $M$ the topological interior and the boundary of a set $M \subset Y$, and $M$ is said to be solid if int $M \neq \emptyset$. The nonnegative orthant of $\mathbb{R}^{p}$ is denoted by $\mathbb{R}_{+}^{p}$. In addition, $\mathbb{R}_{+}:=\mathbb{R}_{+}^{1}$.

The effective domain of an extended real function $g: X \rightarrow \mathbb{R} \cup\{+\infty\}$ is denoted by dom $g$, i.e., $\operatorname{dom} g:=\{x \in X: g(x)<+\infty\}$. Moreover, recall that a function $g: Y \rightarrow \mathbb{R}$ is said to be $D$-monotone (resp. strictly $D$-monotone) if for each $y_{1}, y_{2} \in Y$,

$$
\begin{aligned}
y_{1} \leq_{D} y_{2} & \Rightarrow g\left(y_{1}\right) \leq g\left(y_{2}\right) \\
\left(\text { resp. } y_{1}<_{D} y_{2}\right. & \left.\Rightarrow g\left(y_{1}\right)<g\left(y_{2}\right)\right) .
\end{aligned}
$$

Throughout, the ordering cone $D$ is supposed to be proper (i.e., $\{0\} \neq D \neq Y$ ), closed and solid. In addition, we denote $\tilde{D}:=\operatorname{int} D \cup\{0\}$. It is clear that $\tilde{D}$ is a proper convex cone and $\tilde{D} \backslash\{0\}=\operatorname{int} D$.

This work involves Ekeland variational principles for the following vector equilibrium problem:

$$
\text { Find } \hat{x} \in S \text { such that } F(x, \hat{x}) \nless{ }_{D} 0 \text {, for all } x \in S \text {, }
$$

where $F: X \times X \rightarrow Y$ and $\emptyset \neq S \subset X$. The set of all points satisfying the above condition is denoted by $\operatorname{Sol}(F, S, D)$.

Next, two basic mathematical tools required for obtaining the main results of this paper are recalled. The first one is a version of the Ekeland variational principle for real strict-decreasingly lower-semicontinuous functions. The second one is the well-known Gerstewitz scalarization functional.

Definition 2.1. [5] We say that an extended real function $g: X \rightarrow \mathbb{R} \cup\{+\infty\}$ is strict-decreasingly lower-semicontinuous at a point $x_{0} \in \operatorname{dom} g$ if for each convergent sequence $\left(x_{n}\right)$ in $X, x_{n} \rightarrow x_{0}$, such that $\left(g\left(x_{n}\right)\right)$ is strictly decreasing (i.e., $g\left(x_{n+1}\right)<g\left(x_{n}\right)$, for all $n$ ), it follows that $g\left(x_{0}\right) \leq$ $g\left(x_{n}\right)$, for all $n$. 
Theorem 2.1. [5, Theorem 6] Let $g: X \rightarrow \mathbb{R} \cup\{+\infty\}$ be a proper extended real lower bounded strict-decreasingly lower-semicontinuous function. For each $x_{0} \in \operatorname{dom}$, there exists $\hat{x} \in X$ such that

(1) $g(\hat{x})+d\left(\hat{x}, x_{0}\right) \leq g\left(x_{0}\right)$.

(2) $g(x)+d(x, \hat{x})>g(\hat{x})$, for all $x \in X \backslash\{\hat{x}\}$.

Let $q \in Y \backslash\{0\}$. The so-called Gerstewitz functional $\varphi_{D}^{q}: Y \rightarrow \mathbb{R} \cup\{ \pm \infty\}$ is defined as follows:

$$
\varphi_{D}^{q}(y):=\left\{\begin{array}{cl}
+\infty, & \text { if } y \notin \mathbb{R} q-D \\
\inf \{t \in \mathbb{R}: y \in t q-D\}, & \text { otherwise. }
\end{array}\right.
$$

The next proposition collects the basic properties of this functional when the vector $q$ belongs to the topological interior of $D$ (see $[12,18]$ ).

Proposition 2.1. Suppose that $q \in$ int $D$. Then, the following assertions hold true:

(1) $\varphi_{D}^{q}$ is finite-valued, (i.e., $\varphi_{D}^{q}: Y \rightarrow \mathbb{R}$ ), continuous, subadditive and positively homogeneous.

(2) For each $y \in Y$ and $t \in \mathbb{R}$ it follows that $\varphi_{D}^{q}(y+t q)=\varphi_{D}^{q}(y)+t$.

(3) For each $t \in \mathbb{R}$ we have that

$$
\begin{aligned}
& \left\{y \in Y: \varphi_{D}^{q}(y) \leq t\right\}=t q-D, \\
& \left\{y \in Y: \varphi_{D}^{q}(y)<t\right\}=t q-\operatorname{int} D, \\
& \left\{y \in Y: \varphi_{D}^{q}(y)=t\right\}=t q-\operatorname{bd} D .
\end{aligned}
$$

(4) $\varphi_{D}^{q}$ is D-monotone and strictly $\tilde{D}$-monotone.

\section{MAIN RESUltS}

Consider a function $f: X \rightarrow Y$ and $q \in \operatorname{int} D$. Next, the boundedness from below and the strict-decreasingly lower-semicontinuity of the scalarization $\varphi_{D}^{q} \circ f: X \rightarrow \mathbb{R}$ are characterized by certain boundedness from below and strict-decreasingly lower-semicontinuity properties of the vector function $f$.

The following bounded from below concept for sets in ordered linear spaces is due to Hernández and Rodríguez-Marín [16, Definition 2.15].

Definition 3.1. A nonempty set $M \subset Y$ is said to be $D$-proper if $M+D \neq Y$.

Lemma 3.1. Let $q \in \operatorname{int} D$. A nonempty set $M \subset Y$ is D-proper if and only if there exists $m \in \mathbb{R}$ such that $(M+m q) \cap(-D)=\emptyset$.

Proof. Let us check first the sufficient condition. Then, assume that

$$
(M+m q) \cap(-D)=\emptyset
$$

for some $m \in \mathbb{R}$. Since $D$ is a convex cone, it follows that $D+D=D$. Thus, assertion (3.1) is equivalent to the next one:

$$
(M+D+m q) \cap(-D)=\emptyset .
$$

Reasoning by contradiction, suppose that $M$ is not $D$-proper. Then, $M+D=Y$ and so

$$
-D=Y \cap(-D)=(Y+m q) \cap(-D)=(M+D+m q) \cap(-D),
$$

that is contrary to assertion (3.2). Therefore, the sufficient condition holds true. 
Reciprocally, assume that $M$ is $D$-proper. Reasoning again by contradiction let us suppose that

$$
(M+t q) \cap(-D) \neq \emptyset, \quad \forall t \in \mathbb{R} .
$$

Let $y$ be an arbitrary point of $Y$. It follows that $y \in M+D$. Indeed, as $q \in \operatorname{int} D$ there exists $s>0$ such that $q+s y \in D$. Thus, as $D$ is a cone we deduce that

$$
y \in-(1 / s) q+D
$$

By applying (3.3) to $t=1 / s$, we have that $(M+(1 / s) q) \cap(-D) \neq \emptyset$ and it follows that

$$
-(1 / s) q \in M+D \text {. }
$$

Therefore, as $D+D=D$, by statements (3.4) and (3.5), we have that

$$
y \in-(1 / s) q+D \in M+D+D=M+D .
$$

Then, since $y$ is an arbitrary element of $Y$, the equality $Y=M+D$ follows, which is a contradiction because $M$ is $D$-proper. Thus, (3.3) is not true and there exists $m \in \mathbb{R}$ such that $(M+m q) \cap(-D)=\emptyset$. This finishes the proof.

Let us notice from Lemma 3.1 and the results in [22, Section 3] that the notion of $D$ properness becomes a really general lower boundedness concept for vector functions when it is applied to their image set and the ordering cone is solid.

Theorem 3.1. Let $f: X \rightarrow Y$ and $q \in \operatorname{int} D$. The scalar function $\varphi_{D}^{q} \circ f$ is bounded from below if and only if the image set $f(X)$ is D-proper.

Proof. Function $\varphi_{D}^{q} \circ f$ is bounded from below if and only if there exists $m \in \mathbb{R}$ such that

$$
\left(\varphi_{D}^{q} \circ f\right)(x)>-m, \quad \forall x \in X .
$$

By applying assertion (2.1), we see that statement (3.6) is equivalent to the next one:

$$
f(x) \notin-m q-D, \quad \forall x \in X,
$$

i.e., $(f(X)+m q) \cap(-D)=\emptyset$. Therefore, by Lemma 3.1, we see that $\varphi_{D}^{q} \circ f$ is bounded from below if and only if the image set $f(X)$ is $D$-proper and the proof finishes.

Definition 3.2. Consider $q \in \operatorname{int} D$. A sequence $\left(y_{n}\right)$ in $Y$ is said to be strictly $q$-decreasing if

$$
t \in \mathbb{R}, y_{n} \in t q-D \Rightarrow y_{n+1} \in \operatorname{int}(t q-D) .
$$

Notice that assertion (3.7) can be rewritten as follows:

$$
t \in \mathbb{R}, y_{n} \leq_{D} t q \Rightarrow y_{n+1}<_{\tilde{D}} t q .
$$

Lemma 3.2. Consider $q \in \operatorname{int} D$ and a sequence $\left(y_{n}\right)$ in $Y$. The following assertions are equivalent:

(1) The sequence of real numbers $\left(\varphi_{D}^{q}\left(y_{n}\right)\right)$ is strictly decreasing.

(2) $y_{n+1} \in \operatorname{int}\left(\varphi_{D}^{q}\left(y_{n}\right) q-D\right)$, for all $n \in \mathbb{N}$.

(3) The sequence $\left(y_{n}\right)$ is strictly q-decreasing. 
Proof. Let $\left(y_{n}\right)$ be a sequence in $Y$. By part (2) of Proposition 2.1 and statement (2.2), we see that

$$
\begin{aligned}
\varphi_{D}^{q}\left(y_{n+1}\right)<\varphi_{D}^{q}\left(y_{n}\right) & \Longleftrightarrow \varphi_{D}^{q}\left(y_{n+1}-\varphi_{D}^{q}\left(y_{n}\right) q\right)<0 \\
& \Longleftrightarrow y_{n+1}-\varphi_{D}^{q}\left(y_{n}\right) q \in-\operatorname{int} D \\
& \Longleftrightarrow y_{n+1} \in \operatorname{int}\left(\varphi_{D}^{q}\left(y_{n}\right) q-D\right) .
\end{aligned}
$$

Therefore, statements (1) and (2) above are equivalent.

Next, let us check that statement (2) implies statement (3). Consider and arbitrary $n \in \mathbb{N}$ and a real number $t$ such that $y_{n} \in t q-D$. By the definition of the function $\varphi_{D}^{q}$ it is clear that $\varphi_{D}^{q}\left(y_{n}\right) \leq t$. Then, from assertion (2) we deduce that

$$
y_{n+1} \in \varphi_{D}^{q}\left(y_{n}\right) q-\operatorname{int} D=t q+\left(\varphi_{D}^{q}\left(y_{n}\right)-t\right) q-\operatorname{int} D \subset t q-\operatorname{int} D,
$$

since $q \in \operatorname{int} D$ and $\tilde{D}+\operatorname{int} D=\operatorname{int} D$. Then part (3) holds true whenever part (2) is fulfilled.

Finally, part (3) implies part (1). Indeed, consider $n \in \mathbb{N}$. By statement (2.1) we see that $y_{n} \in$ $\varphi_{D}^{q}\left(y_{n}\right) q-D$. Then, by applying the definition of strictly $q$-decreasing sequence to $t=\varphi_{D}^{q}\left(y_{n}\right)$ we deduce that

$$
y_{n+1} \in \operatorname{int}\left(\varphi_{D}^{q}\left(y_{n}\right) q-D\right)=\varphi_{D}^{q}\left(y_{n}\right) q-\operatorname{int} D .
$$

By this condition and statement (2.2) it follows that $\varphi_{D}^{q}\left(y_{n+1}\right)<\varphi_{D}^{q}\left(y_{n}\right)$. Thus, assertion (1) follows and the proof is completed.

As a result of Lemma 3.2, we deduce that the notion of strictly $q$-decreasing sequence reduces to the usual concept of strictly decreasing sequence of real numbers.

Corollary 3.1. Assume that $Y=\mathbb{R}$ and $D=\mathbb{R}_{+}$. Consider $c>0$. Then, a sequence $\left(r_{n}\right)$ of real numbers is strictly c-decreasing if and only if $r_{n+1}<r_{n}$, for all $n$.

Proof. Let $c>0$. By Lemma 3.2, a sequence $\left(r_{n}\right)$ in $\mathbb{R}$ is strictly $c$-decreasing if and only if the sequence $\left(\varphi_{\mathbb{R}_{+}}^{c}\left(r_{n}\right)\right)$ of real numbers is strictly decreasing. It is easy to check that $\varphi_{\mathbb{R}_{+}}^{c}(r)=r / c$, for all $r \in \mathbb{R}$. Therefore, $\left(\varphi_{\mathbb{R}_{+}}^{c}\left(r_{n}\right)\right)$ is strictly decreasing if and only if $r_{n+1} / c<r_{n} / c$, for all $n$, i.e., if and only if $\left(r_{n}\right)$ is a strictly decreasing sequence of real numbers, and the proof is completed.

Definition 3.3. Consider $q \in \operatorname{int} D$. A function $f: X \rightarrow Y$ is said to be strict $q$-decreasingly $D$-lower-semicontinuous at a point $x_{0} \in X$ if for each convergent sequence $\left(x_{n}\right)$ in $X, x_{n} \rightarrow x_{0}$, such that $\left(f\left(x_{n}\right)\right)$ is strictly $q$-decreasing, it follows that for each $n$,

$$
t \in \mathbb{R}, f\left(x_{n}\right) \in t q-D \Rightarrow f\left(x_{0}\right) \in t q-D .
$$

It is said that $f$ is strict $q$-decreasingly $D$-lower-semicontinuous if it is strict $q$-decreasingly $D$-lower-semicontinuous at each point $x \in X$.

Although the proof of the following result is similar to the proof of Lemma 3.2, we give it for the convenience of the reader.

Proposition 3.1. Consider $q \in$ int $D$ and two points $y_{1}, y_{2}$ in $Y$. The next statements are equivalent:

(1) $\varphi_{D}^{q}\left(y_{1}\right) \leq \varphi_{D}^{q}\left(y_{2}\right)$.

(2) $y_{1} \leq_{D} \varphi_{D}^{q}\left(y_{2}\right) q$. 
(3) $t \in \mathbb{R}, y_{2} \in t q-D \Rightarrow y_{1} \in t q-D$.

Proof. By part (2) of Proposition 2.1 and statement (2.1), it follows that

$$
\begin{aligned}
\varphi_{D}^{q}\left(y_{1}\right) \leq \varphi_{D}^{q}\left(y_{2}\right) & \Longleftrightarrow \varphi_{D}^{q}\left(y_{1}-\varphi_{D}^{q}\left(y_{2}\right) q\right) \leq 0 \\
& \Longleftrightarrow y_{1}-\varphi_{D}^{q}\left(y_{2}\right) q \in-D \\
& \Longleftrightarrow y_{1} \leq{ }_{D} \varphi_{D}^{q}\left(y_{2}\right) q
\end{aligned}
$$

and so parts (1) and (2) above are equivalent.

Suppose that part (2) holds true and consider $t \in \mathbb{R}$ such that $y_{2} \in t q-D$. By the definition of the function $\varphi_{D}^{q}$ we see that $\varphi_{D}^{q}\left(y_{2}\right) \leq t$. Then, by part (2), we deduce that

$$
y_{1} \in \varphi_{D}^{q}\left(y_{2}\right) q-D=t q+\left(\varphi_{D}^{q}\left(y_{2}\right)-t\right) q-D \subset t q-D
$$

as $\left(t-\varphi_{D}^{q}\left(y_{2}\right)\right) q \in \tilde{D}$ and $\tilde{D}+D=D$. Thus, part (3) holds true.

Finally, assume that part (3) is fulfilled. By statement (2.1), we see that $y_{2} \in \varphi_{D}^{q}\left(y_{2}\right) q-D$. Then, by part (3), we have that $y_{1} \in \varphi_{D}^{q}\left(y_{2}\right) q-D$ and from (2.1) again we deduce that $\varphi_{D}^{q}\left(y_{1}\right) \leq$ $\varphi_{D}^{q}\left(y_{2}\right)$. Thus, part (3) implies part (1) and the proof is completed.

Theorem 3.2. Let $f: X \rightarrow Y, q \in \operatorname{int} D$ and $x_{0} \in X$. The scalarization $\varphi_{D}^{q} \circ f$ is strict-decreasingly lower-semicontinuous at $x_{0}$ if and only if $f$ is strict $q$-decreasingly D-lower-semicontinuous at $x_{0}$.

Proof. Assume that $\varphi_{D}^{q} \circ f$ is strict-decreasingly lower-semicontinuous at $x_{0}$. In order to check that $f$ is strict $q$-decreasingly $D$-lower-semicontinuous at $x_{0}$, we take a convergent sequence $\left(x_{n}\right)$ in $X, x_{n} \rightarrow x_{0}$ such that the sequence $\left(f\left(x_{n}\right)\right)$ is strictly $q$-decreasing.

By Lemma 3.2, we see that the sequence $\left(\varphi_{D}^{q}\left(f\left(x_{n}\right)\right)\right)$ of real numbers is strictly decreasing. Since $\varphi_{D}^{q} \circ f$ is strict-decreasingly lower semicontinuous at $x_{0}$, it follows that $\left(\varphi_{D}^{q} \circ f\right)\left(x_{0}\right) \leq$ $\left(\varphi_{D}^{q} \circ f\right)\left(x_{n}\right)$, for all $n \in \mathbb{N}$. Then, by Proposition 3.1, we deduce that condition (3.8) holds true. Thus, $f$ is strict $q$-decreasingly $D$-lower-semicontinuous at $x_{0}$ and the necessary condition is stated.

Reciprocally, assume that $f$ is strict $q$-decreasingly $D$-lower-semicontinuous at $x_{0}$ and consider a convergent sequence $\left(x_{n}\right)$ in $X, x_{n} \rightarrow x_{0}$ such that the sequence $\left(\left(\varphi_{D}^{q} \circ f\right)\left(x_{n}\right)\right)$ of real numbers is strictly decreasing.

By Lemma 3.2, it follows that the sequence $\left(f\left(x_{n}\right)\right)$ in $Y$ is strictly $q$-decreasing. As $f$ is strict $q$-decreasingly $D$-lower-semicontinuous at $x_{0}$, we see that assertion (3.8) holds true. Then, by Proposition 3.1, we have that $\left(\varphi_{D}^{q} \circ f\right)\left(x_{0}\right) \leq\left(\varphi_{D}^{q} \circ f\right)\left(x_{n}\right)$, for all $n \in \mathbb{N}$. Thus, the scalarization $\varphi_{D}^{q} \circ f$ is strict-decreasingly lower-semicontinuous at $x_{0}$ and the proof is completed.

Remark 3.1. As a result of Theorem 3.2, it follows that the notion of strict $q$-decreasingly $D$ lower-semicontinuous vector function encompasses the corresponding scalar notion recalled in Definition 2.1. Indeed, by Theorem 3.2, a function $f: X \rightarrow \mathbb{R}$ is strict 1-decreasingly $\mathbb{R}_{+}$-lower semicontinuous at $x_{0} \in X$ if and only if $\varphi_{\mathbb{R}_{+}}^{1} \circ f$ is strict-decreasingly lower-semicontinuous at $x_{0}$. As $\varphi_{\mathbb{R}_{+}}^{1}(r)=r$, for all $r \in \mathbb{R}$, this last assertion means that $f$ is strict-decreasingly lowersemicontinuous.

Next, a recent lower semicontinuity concept for vector functions mapping to a finite dimensional space is recalled (see [15, Definition 3]). It was applied to the particular case when the convex cone $H$ is solid and $b \in \operatorname{int} H$ (see, for instance, [15, Theorem 3]). 
Definition 3.4. Let $H \subset \mathbb{R}^{p}$ be a closed convex cone and $b \in \mathbb{R}^{p} \backslash\{0\}$. A function $f: X \rightarrow \mathbb{R}^{p}$ is called $(b, H)$-quasi lower semicontinuous from above at $x_{0} \in X$ if for each $r \in \mathbb{R}$ and $x_{n} \rightarrow x_{0}$, from $f\left(x_{n}\right)+r b \leq_{H} 0$ and $f\left(x_{n}\right) \mathbb{Z}_{H} f\left(x_{n+1}\right)$ for all $n$, it follows that $f\left(x_{0}\right)+r b \leq_{H} 0$.

The concept of strict $q$-decreasingly $D$-lower-semicontinuity introduced in Definition 3.3 is more general than the notion of $(b, H)$-quasi lower semicontinuity from above provided that $b \in \operatorname{int} H$.

Lemma 3.3. Consider $y_{1}, y_{2} \in Y$ and $q \in \operatorname{int} D$. If $y_{2}<_{\tilde{D}} \varphi_{D}^{q}\left(y_{1}\right) q$, then $y_{1} \not_{D} y_{2}$.

Proof. Reasoning by contradiction, suppose that $y_{2}<_{\tilde{D}} \varphi_{D}^{q}\left(y_{1}\right) q$ and $y_{1} \leq_{D} y_{2}$. From this last assertion and part (4) of Proposition 2.1, we have that $\varphi_{D}^{q}\left(y_{1}\right) \leq \varphi_{D}^{q}\left(y_{2}\right)$. Moreover, by the first assertion, part (2) of Proposition 2.1 and statement (2.2), we see that $\varphi_{D}^{q}\left(y_{2}\right)<\varphi_{D}^{q}\left(y_{1}\right)$, which is a contradiction. Thus, $y_{1} \not_{D} y_{2}$ whenever $y_{2}<_{\tilde{D}} \varphi_{D}^{q}\left(y_{1}\right) q$ and the proof finishes.

Theorem 3.3. Let $H \subset \mathbb{R}^{p}$ be a proper solid convex cone and consider $q \in \operatorname{int} H$ and $x_{0} \in$ $X$. If $f: X \rightarrow \mathbb{R}^{p}$ is $(q, H)$-quasi lower semicontinuous from above at $x_{0}$, then it is strict $q$ decreasingly $H$-lower-semicontinuous at $x_{0}$.

Proof. Let $\left(x_{n}\right)$ be a convergent sequence in $X, x_{n} \rightarrow x_{0}$ such that $\left(f\left(x_{n}\right)\right)$ is strictly $q$-decreasing. Let us check that

$$
f\left(x_{0}\right) \leq_{H} \varphi_{D}^{q}\left(f\left(x_{n}\right)\right) q, \quad \forall n \in \mathbb{N} .
$$

Indeed, consider an arbitrary $k \in \mathbb{N}$ and the sequence $\left(u_{n}\right)$ in $X, u_{n}:=x_{n+k}$, for all $n \in \mathbb{N}$. It is obvious that $u_{n} \rightarrow x_{0}$. Moreover, by part (2) of Lemma 3.2, we see that $f\left(u_{n+1}\right)<_{\tilde{H}} \varphi_{H}^{q}\left(f\left(u_{n}\right)\right) q$. By Lemma 3.3, we deduce that $f\left(u_{n}\right) \not_{H} f\left(u_{n+1}\right)$, for all $n \in \mathbb{N}$.

In addition, as $\left(f\left(x_{n}\right)\right)$ is strictly $q$-decreasing, by part (1) of Lemma 3.2, we see that the sequence $\left(\varphi_{H}^{q}\left(f\left(x_{n}\right)\right)\right)$ is strictly decreasing. In particular, $\varphi_{H}^{q}\left(f\left(u_{n}\right)\right) \leq \varphi_{H}^{q}\left(f\left(x_{k}\right)\right)$, for all $n$. By part (2) of Proposition 3.1, we have that $f\left(u_{n}\right)-\varphi_{H}^{q}\left(f\left(x_{k}\right)\right) q \leq_{H} 0$, for all $n$. Let $r:=$ $-\varphi_{H}^{q}\left(f\left(x_{k}\right)\right)$. Since $f$ is $(q, H)$-quasi lower semicontinuous from above at $x_{0}$, it follows that $f\left(x_{0}\right)+r q \leq_{H}$ 0, i.e., $f\left(x_{0}\right) \leq_{H} \varphi_{H}^{q}\left(f\left(x_{k}\right)\right) q$. As $k$ is arbitrary, statement (3.9) is proved.

Finally, this statement and part (3) of Proposition 3.1 imply that assertion (3.8) holds true for all $n \in \mathbb{N}$. It follows that $f$ is strict $q$-decreasingly $H$-lower-semicontinuous at $x_{0}$ and the proof finishes.

Remark 3.2. The reciprocal implication of Theorem 3.3 is not true in general, as it is showed in the following example. Let $X=\mathbb{R}, p=2, H=\mathbb{R}_{+}^{2}, q=(1,1)$ and the function $f: \mathbb{R} \rightarrow \mathbb{R}^{2}$, $f(s)=(1 / s, 0)$ if $s<0$ and $f(s)=(0,1+s)$ otherwise.

It is easy to check that $\varphi_{H}^{q}(y)=\max \left\{y_{1}, y_{2}\right\}$, for all $y=\left(y_{1}, y_{2}\right) \in \mathbb{R}^{2}$. Thus, $\left(\varphi_{H}^{q} \circ f\right)(s)=$ 0 if $s<0$ and $\left(\varphi_{H}^{q} \circ f\right)(s)=1+s$ otherwise. This function is strict-decreasingly lower semicontinuous at $s=0$ and by Theorem 3.2 we deduce that $f$ is strict $q$-decreasingly $H$ lower-semicontinuous at $s=0$. Indeed, consider a sequence $\left(s_{n}\right)$ in $\mathbb{R}, s_{n} \rightarrow 0$ such that $\left(\left(\varphi_{H}^{q} \circ f\right)\left(s_{n}\right)\right)$ is strictly decreasing. It follows that $\left(s_{n}\right) \subset(0,+\infty)$ and it is strictly decreasing. Then, $\left(\varphi_{H}^{q} \circ f\right)(0)<\left(\varphi_{H}^{q} \circ f\right)\left(s_{n}\right)$ for all $n \in \mathbb{N}$ and we have that $\varphi_{H}^{q} \circ f$ is strict-decreasingly lower-semicontinuous at $s=0$.

However, $f$ is not $(q, H)$-quasi lower semicontinuous from above at 0 . Indeed, consider $r=0$ and the sequence $\left(s_{n}\right) \subset \mathbb{R}, s_{n}=-1 / n$ for all $n \in \mathbb{N}$. We have that $s_{n} \rightarrow 0$ and $f\left(s_{n}\right)=(-n, 0)$. Thus, $f\left(s_{n}\right) \not_{H} f\left(s_{n+1}\right)$, for all $n \in \mathbb{N}$. Moreover, $f\left(s_{n}\right) \leq_{H} 0$ for all $n \in \mathbb{N}$, but $f(0) \not_{H}(0,0)$. 
Now, we are ready to give our main results.

Theorem 3.4. Consider a function $f: X \rightarrow Y$ and $q \in \operatorname{int} D$ such that $f(X)$ is D-proper and $f$ is strict q-decreasingly D-lower-semicontinuous. Then, for each $x_{0} \in X$ there exists $\hat{x} \in X$ such that

(1) $\varphi_{D}^{q}(f(\hat{x}))+d\left(\hat{x}, x_{0}\right) \leq \varphi_{D}^{q}\left(f\left(x_{0}\right)\right)$.

(2) $\varphi_{D}^{q}(f(x))+d(x, \hat{x})>\varphi_{D}^{q}(f(\hat{x}))$, for all $x \in X \backslash\{\hat{x}\}$.

Proof. Consider an arbitrary point $x_{0} \in X$ and the function $g:=\varphi_{D}^{q} \circ f$. By Theorems 3.1 and 3.2, we deduce that the function $g$ is bounded from below and strict-decreasingly lowersemicontinuous. Then, the result follows by applying Theorem 2.1.

An equivalent formulation of Theorem 3.4 is obtained in Corollary 3.2 below. For this aim, the next simple lemma is needed.

Lemma 3.4. Let $y \in Y, q \in \operatorname{int} D$ and $r, s \in \mathbb{R}$. We have that

$$
\varphi_{D}^{q}(y)+r \leq s \Longleftrightarrow y+r q \leq_{D} s q .
$$

Proof. By part (2) of Proposition 2.1 and statement (2.1) we see that

$$
\begin{aligned}
\varphi_{D}^{q}(y)+r \leq s & \Longleftrightarrow \varphi_{D}^{q}(y+(r-s) q) \leq 0 \\
& \Longleftrightarrow y+(r-s) q \in-D \\
& \Longleftrightarrow y+r q \leq{ }_{D} s q
\end{aligned}
$$

and the proof is completed.

Next corollary is an obvious consequence of Theorem 3.4 and Lemma 3.4.

Corollary 3.2. Consider a function $f: X \rightarrow Y$ and $q \in \operatorname{int} D$ such that $f(X)$ is D-proper and $f$ is strict $q$-decreasingly $D$-lower-semicontinuous. Then, for each $x_{0} \in X$ there exists $\hat{x} \in X$ such that

(1) $f(\hat{x})+d\left(\hat{x}, x_{0}\right) q \leq_{D} \varphi_{D}^{q}\left(f\left(x_{0}\right)\right) q$.

(2) $f(x)+d(x, \hat{x}) q \not \mathbb{L}_{D} \varphi_{D}^{q}(f(\hat{x})) q$, for all $x \in X \backslash\{\hat{x}\}$.

Next, a formulation of the Ekeland variational principle that takes into account approximate solutions of problem (VEP) is stated. It is based on the following notion of weak approximate solution of problem (VEP), which extends to vector equilibrium problems the concept of weak approximate efficiency introduced in [13, Section 4] for a vector optimization problem.

Definition 3.5. Consider a nonempty set $C \subset Y$ such that $C \cap(-\operatorname{int} D)=\emptyset$ and $\varepsilon>0$. A point $\hat{x} \in S$ is said to be a weak $(C, \varepsilon)$-solution of problem (VEP), denoted by $\operatorname{WSol}(F, S, C, \varepsilon)$, if $F(x, \hat{x}) \notin-\varepsilon C-\operatorname{int} D=\emptyset$, for all $x \in S$.

Remark 3.3. (1) Condition $C \cap(-$ int $D)=\emptyset$ above is needed in order to obtain suitable sets of weak approximate solutions (see [13, Remark 2.4]). For instance, consider problem (VEP) with the following data: $X=Y=\mathbb{R}^{2}, F\left(\left(x_{1}, x_{2}\right),\left(y_{1}, y_{2}\right)\right)=\left(x_{1}-y_{1}, x_{2}-y_{2}\right)$, for all $\left(x_{1}, x_{2}\right),\left(y_{1}, y_{2}\right) \in X, S=\mathbb{R}_{+}\{(1,0)\}, D=\left\{\left(y_{1}, y_{2}\right) \in \mathbb{R}^{2}: y_{1} \geq 0\right\}$, and $C=$ $\{(-1,0)\}$. It is easy to check that $\operatorname{Sol}(F, S, D)=\{(0,0)\}$ and $\operatorname{WSol}(F, S, C, \varepsilon)=\emptyset$, for all $\varepsilon>0$. Therefore, the sets $\operatorname{WSol}(F, S, C, \varepsilon)$ of weak approximate solutions are not suitable as the exact solution $(0,0)$ is not an approximate solution for any error $\varepsilon>0$. 
(2) Let $q \in \operatorname{int} D$. Since int $D \subset-s q+\operatorname{int} D$, for all $s>0$, a stronger condition is $C \cap(s q-$ $\operatorname{int} D)=\emptyset$, for some $s>0$, which is equivalent to $\inf _{c \in C} \varphi_{D}^{q}(c)>0$ (see part (a) of [15, Remark 6]).

We denote

$$
\begin{aligned}
\mathscr{C} & :=\{\emptyset \neq C \subset Y: C \cap(-\operatorname{int} D)=\emptyset\}, \\
\mathscr{C}_{q} & :=\bigcup_{s>0}\{\emptyset \neq C \subset Y: C \cap(s q-\operatorname{int} D)=\emptyset\}
\end{aligned}
$$

and $\delta_{q}^{C}:=\inf _{c \in C} \varphi_{D}^{q}(c)$, for each $q \in \operatorname{int} D$ and $\emptyset \neq C \subset Y$. In addition, for each proper real function $g: X \rightarrow \mathbb{R} \cup\{+\infty\}, \emptyset \neq S \subset X$ and $\delta \geq 0$,

$$
\operatorname{argmin}(g, S, \delta):=\left\{x_{0} \in \operatorname{dom} g: g\left(x_{0}\right)-\delta \leq g(x), \forall x \in S\right\} .
$$

The existence of weak $(C, \varepsilon)$-solutions implies that problem (VEP) is bounded from below in a certain sense. To be precise, we have the next result.

Proposition 3.2. Consider problem (VEP), $C \in \mathscr{C}$ and $\varepsilon>0$. If $x_{0} \in W \operatorname{Sol}(F, S, C, \varepsilon)$, then $F\left(S, x_{0}\right)$ is a D-proper set.

Proof. Since $x_{0} \in \mathrm{WSol}(F, S, C, \varepsilon)$, we have that $F\left(S, x_{0}\right) \cap(-\varepsilon C-\operatorname{int} D)=\emptyset$. As $D+\operatorname{int} D=$ int $D$, this condition is equivalent to the next one:

$$
\left(F\left(S, x_{0}\right)+D\right) \cap(-\varepsilon C-\operatorname{int} D)=\emptyset .
$$

Thus, $F\left(S, x_{0}\right)+D \neq Y$ and $F\left(S, x_{0}\right)$ is a $D$-proper set.

Next, a necessary condition by scalarization for weak $(C, \varepsilon)$-solutions of problem (VEP) is obtained. Then, an Ekeland variational principle for approximate solutions of problem (VEP) follows from this condition and Theorem 3.4.

Proposition 3.3. Consider problem (VEP), $C \in \mathscr{C}, \varepsilon>0$, and $q \in \operatorname{int} D$. If $x_{0} \in W \operatorname{Wol}(F, S, C, \varepsilon)$ and $F\left(x_{0}, x_{0}\right) \leq{ }_{D} 0$, then $x_{0} \in \operatorname{argmin}\left(\left(\varphi_{D}^{q} \circ F\right)\left(\cdot, x_{0}\right), S, \varepsilon \delta_{q}^{C}\right)$.

Proof. Since $x_{0} \in \mathrm{WSol}(F, S, C, \varepsilon)$, it follows that $F\left(S, x_{0}\right) \cap(-\varepsilon C-\operatorname{int} D)=\emptyset$. Thus, $\left(F\left(S, x_{0}\right)+\right.$ $\varepsilon C) \cap(-\mathrm{int} D)=\emptyset$ and by statement (2.2) we deduce that $\varphi_{D}^{q}\left(F\left(x, x_{0}\right)+\varepsilon c\right) \geq 0$, for all $x \in S$ and $c \in C$. From assertion (1) of Proposition 2.1 we know that $\varphi_{D}^{q}$ is subadditive and positively homogeneous. Therefore,

$$
0 \leq \varphi_{D}^{q}\left(F\left(x, x_{0}\right)+\varepsilon c\right) \leq\left(\varphi_{D}^{q} \circ F\right)\left(x, x_{0}\right)+\varepsilon \varphi_{D}^{q}(c), \quad \forall x \in S, c \in C .
$$

As $F\left(x_{0}, x_{0}\right) \leq_{D} 0$, by statement (2.1) of Proposition 2.1 it follows that $\left(\varphi_{D}^{q} \circ F\right)\left(x_{0}, x_{0}\right) \leq 0$. Then, by taking $x=x_{0}$ above, we have that $\varepsilon \varphi_{D}^{q}(c) \geq 0$, for all $c \in C$. Thus, $\varepsilon \delta_{q}^{C} \geq 0$ and assertion (3.10) implies that

$$
\left(\varphi_{D}^{q} \circ F\right)\left(x_{0}, x_{0}\right) \leq\left(\varphi_{D}^{q} \circ F\right)\left(x, x_{0}\right)+\varepsilon \delta_{q}^{C}, \quad \forall x \in S,
$$

i.e., $x_{0} \in \operatorname{argmin}\left(\left(\varphi_{D}^{q} \circ F\right)\left(\cdot, x_{0}\right), S, \varepsilon \delta_{q}^{C}\right)$ and the proof is completed.

Theorem 3.5. Consider problem (VEP) with $S=X, C \in \mathscr{C}, \varepsilon>0, x_{0} \in W \operatorname{Sol}(F, X, C, \varepsilon)$ and $q \in \operatorname{int} D$. Suppose that $F\left(\cdot, x_{0}\right): X \rightarrow Y$ is strict $q$-decreasingly D-lower-semicontinuous and $F\left(x_{0}, x_{0}\right) \leq{ }_{D} 0$. Then, there exists $\hat{x} \in X$ such that

(1) $\varphi_{D}^{q}\left(F\left(\hat{x}, x_{0}\right)\right)+d\left(\hat{x}, x_{0}\right) \leq 0$. 
(2) $d\left(\hat{x}, x_{0}\right) \leq \varepsilon \delta_{q}^{C}$.

(3) $\varphi_{D}^{q}\left(F\left(x, x_{0}\right)\right)+d(x, \hat{x})>\varphi_{D}^{q}\left(F\left(\hat{x}, x_{0}\right)\right)$, for all $x \in X \backslash\{\hat{x}\}$.

If in addition $C \in \mathscr{C}_{q}$, then, for each $\lambda>0$, there exists $\hat{x}_{\lambda} \in X$ such that

(4) $\varphi_{D}^{q}\left(F\left(\hat{x}_{\lambda}, x_{0}\right)\right)+\frac{\varepsilon \delta_{q}^{C}}{\lambda} d\left(\hat{x}_{\lambda}, x_{0}\right) \leq 0$.

(5) $d\left(\hat{x}_{\lambda}, x_{0}\right) \leq \lambda$.

(6) $\varphi_{D}^{q}\left(F\left(x, x_{0}\right)\right)+\frac{\varepsilon \delta_{q}^{C}}{\lambda} d\left(x, \hat{x}_{\lambda}\right)>\varphi_{D}^{q}\left(F\left(\hat{x}_{\lambda}, x_{0}\right)\right)$, for all $x \in X \backslash\left\{\hat{x}_{\lambda}\right\}$.

Proof. Let $f_{x_{0}}: X \rightarrow \mathbb{R}$ be the function $f_{x_{0}}(x):=F\left(x, x_{0}\right)$, for all $x \in X$. By Proposition 3.2, we have that $f_{x_{0}}(X)$ is a $D$-proper set. Then, by applying Theorem 3.4, we deduce that there exists a point $\hat{x} \in X$ satisfying

(1) $\varphi_{D}^{q}\left(f_{x_{0}}(\hat{x})\right)+d\left(\hat{x}, x_{0}\right) \leq \varphi_{D}^{q}\left(f_{x_{0}}\left(x_{0}\right)\right)$.

(2) $\varphi_{D}^{q}\left(f_{x_{0}}(x)\right)+d(x, \hat{x})>\varphi_{D}^{q}\left(f_{x_{0}}(\hat{x})\right)$, for all $x \in X \backslash\{\hat{x}\}$.

As a result, the third assertion of the theorem holds true by condition (2) above. Moreover, statement (2.1) implies that $\varphi_{D}^{q}\left(f_{x_{0}}\left(x_{0}\right)\right) \leq 0$, since $f_{x_{0}}\left(x_{0}\right) \leq{ }_{D} 0$. Therefore, the first statement of the theorem follows from assertion (1) above.

In addition, by Proposition 3.3 and condition (1) above, we see that

$$
d\left(\hat{x}, x_{0}\right) \leq \varphi_{D}^{q}\left(F\left(x_{0}, x_{0}\right)\right)-\varphi_{D}^{q}\left(F\left(\hat{x}, x_{0}\right)\right) \leq \varepsilon \delta_{q}^{C}
$$

and the second statement of the theorem is proved.

Finally, by the second part of Remark 3.3, we deduce that $C \in \mathscr{C}$ and $\delta_{q}^{C}>0$ provided that $C \in \mathscr{C}_{q}$. Thus, whenever this condition holds true and for each $\lambda>0$, parts (4)-(6) follow by applying parts (1)-(3) to the distance $d^{\prime}: X \times X \rightarrow \mathbb{R}, d^{\prime}\left(x_{1}, x_{2}\right):=\frac{\varepsilon \delta_{q}^{C}}{\lambda} d\left(x_{1}, x_{2}\right)$, for all $x_{1}, x_{2} \in X$.

Notice that assertions (3) and (6) of the Ekeland variational principles stated in Theorem 3.5 do not involve proper perturbation functions, since the point $x_{0}$ is considered in the term $\varphi_{D}^{q}\left(F\left(x, x_{0}\right)\right)$ instead of the points $\hat{x}$ and $\hat{x}_{\lambda}$, respectively. Next, Ekeland variational principles with proper perturbation functions are obtained.

Definition 3.6. We say that the bifunction $F: X \times X \rightarrow Y$ satisfies the triangle inequality property in $-D$ by $q \in$ int $D$ at a point $x_{0} \in X$ if $F\left(x_{1}, x_{0}\right) \leq_{D} \varphi_{D}^{q}\left(F\left(x_{1}, x_{2}\right)\right) q+\varphi_{D}^{q}\left(F\left(x_{2}, x_{0}\right)\right) q$ provided that the points $x_{1}, x_{2} \in X$ satisfy $F\left(x_{1}, x_{2}\right) \in-D$ and $F\left(x_{2}, x_{0}\right) \in-\operatorname{int} D$.

Remark 3.4. In [15], the following condition was introduced:

$\left(\mathscr{A}_{D}\right)$ If $\left(F\left(x_{1}, x_{2}\right), F\left(x_{2}, x_{3}\right)\right) \in(-D) \times(-D) \backslash\{(0,0)\}$, then $F\left(x_{1}, x_{3}\right) \leq_{D} F\left(x_{1}, x_{2}\right)+F\left(x_{2}, x_{3}\right)$.

The concept introduced in Definition 3.6 is more general than this property. On the one hand, if follows that $F$ satisfies the triangle inequality property in $-D$ by $q$ at $x_{0}$, for all $q \in \operatorname{int} D$ and $x_{0} \in X$, as long as property $\left(\mathscr{A}_{D}\right)$ holds true. Indeed, take an arbitrary $q \in \operatorname{int} D$ and let $x_{0}, x_{1}, x_{2} \in X$ be such that $F\left(x_{1}, x_{2}\right) \in-D$ and $F\left(x_{2}, x_{0}\right) \in-i n t D$. Then, $\left(F\left(x_{1}, x_{2}\right), F\left(x_{2}, x_{0}\right)\right) \in$ $(-D) \times(-D) \backslash\{(0,0)\}$. By condition $\left(\mathscr{A}_{D}\right)$, we have that $F\left(x_{1}, x_{0}\right) \leq_{D} F\left(x_{1}, x_{2}\right)+F\left(x_{2}, x_{0}\right)$. By statement (4) of Proposition 2.1, we see that $\varphi_{D}^{q}\left(F\left(x_{1}, x_{0}\right)\right) \leq \varphi_{D}^{q}\left(F\left(x_{1}, x_{2}\right)+F\left(x_{2}, x_{0}\right)\right)$, which is equivalent to the assertion $F\left(x_{1}, x_{0}\right) \leq_{D} \varphi_{D}^{q}\left(F\left(x_{1}, x_{2}\right)+F\left(x_{2}, x_{0}\right)\right) q$ by Proposition 3.1. Since $\varphi_{D}^{q}$ is subadditive, we deduce

$$
F\left(x_{1}, x_{0}\right) \leq_{D} \varphi_{D}^{q}\left(F\left(x_{1}, x_{2}\right)\right) q+\varphi_{D}^{q}\left(F\left(x_{2}, x_{0}\right)\right) q,
$$


i.e., $F$ fulfills the triangle inequality property in $-D$ by $q$ at $x_{0}$.

On the other hand, consider $X=\mathbb{R}, Y=\mathbb{R}^{2}, D=\mathbb{R}_{+}^{2}$ and $F: \mathbb{R}^{2} \rightarrow \mathbb{R}^{2}, F\left(x_{1}, x_{2}\right)=(1,1)$ if $x_{1}>x_{2}$ and $F\left(x_{1}, x_{2}\right)=(-1,0)$ otherwise. It is easy to check that $F$ satisfies the triangle inequality property in $-D$ by $q$ at $x_{0}$, for all $q \in \operatorname{int} D$ and $x_{0} \in \mathbb{R}$, but it does not fulfill property $\left(\mathscr{A}_{D}\right)$.

Theorem 3.6. Consider problem (VEP) with $S=X, C \in \mathscr{C}, \varepsilon>0, x_{0} \in W \operatorname{Sol}(F, X, C, \varepsilon)$ and $q \in \operatorname{int} D$. Suppose that $F\left(\cdot, x_{0}\right): X \rightarrow Y$ is strict $q$-decreasingly D-lower-semicontinuous, $F$ satisfies the triangle inequality property in $-D$ by $q$ at $x_{0}$ and $F\left(x_{0}, x_{0}\right) \in-\operatorname{bd} D$. Then, there exists $\hat{x} \in X$ such that

(1) $\varphi_{D}^{q}\left(F\left(\hat{x}, x_{0}\right)\right)+d\left(\hat{x}, x_{0}\right) \leq 0$.

(2) $d\left(\hat{x}, x_{0}\right) \leq \varepsilon \delta_{q}^{C}$.

(3) $\varphi_{D}^{q}(F(x, \hat{x}))+d(x, \hat{x})>0$, for all $x \in X \backslash\{\hat{x}\}$.

If in addition $C \in \mathscr{C}_{q}$, then, for each $\lambda>0$, there exists $\hat{x}_{\lambda} \in X$ such that

(4) $\varphi_{D}^{q}\left(F\left(\hat{x}_{\lambda}, x_{0}\right)\right)+\frac{\varepsilon \delta_{q}^{C}}{\lambda} d\left(\hat{x}_{\lambda}, x_{0}\right) \leq 0$.

(5) $d\left(\hat{x}_{\lambda}, x_{0}\right) \leq \lambda$.

(6) $\varphi_{D}^{q}\left(F\left(x, \hat{x}_{\lambda}\right)\right)+\frac{\varepsilon \delta_{q}^{C}}{\lambda} d\left(x, \hat{x}_{\lambda}\right)>0$, for all $x \in X \backslash\left\{\hat{x}_{\lambda}\right\}$.

Proof. It is clear from Theorem 3.5 that there exists a point $\hat{x}$ fulfilling assertions (1), (2) and the next one:

$$
\varphi_{D}^{q}\left(F\left(x, x_{0}\right)\right)+d(x, \hat{x})>\varphi_{D}^{q}\left(F\left(\hat{x}, x_{0}\right)\right), \quad \forall x \in X \backslash\{\hat{x}\} .
$$

If $\hat{x}=x_{0}$, then statement (3) holds true as it coincides with the assertion above (notice that $F\left(x_{0}, x_{0}\right) \in-\mathrm{bd} D$ from the assumptions and then $\varphi_{D}^{q}\left(F\left(x_{0}, x_{0}\right)\right)=0$ by statement (2.3)). Otherwise, suppose reasoning by contradiction that there exists $\bar{x} \in X \backslash\{\hat{x}\}$ such that

$$
\varphi_{D}^{q}(F(\bar{x}, \hat{x}))+d(\bar{x}, \hat{x}) \leq 0 .
$$

Thus, $\varphi_{D}^{q}(F(\bar{x}, \hat{x})) \leq 0$ and by statement (2.1), we deduce that $F(\bar{x}, \hat{x}) \in-D$. Analogously, from part (1), we see that $\varphi_{D}^{q}\left(F\left(\hat{x}, x_{0}\right)\right)<0$, since $\hat{x} \neq x_{0}$. Thus, by statement (2.2) it follows that $F\left(\hat{x}, x_{0}\right) \in-\operatorname{int} D$. Since $F$ satisfies the triangle inequality property in $-D$ by $q$ at $x_{0}$, we deduce that

$$
F\left(\bar{x}, x_{0}\right) \leq_{D} \varphi_{D}^{q}(F(\bar{x}, \hat{x})) q+\varphi_{D}^{q}\left(F\left(\hat{x}, x_{0}\right)\right) q .
$$

By Proposition 3.1 and part (2) of Proposition 2.1, we see that

$$
\varphi_{D}^{q}\left(F\left(\bar{x}, x_{0}\right)\right) \leq \varphi_{D}^{q}(F(\bar{x}, \hat{x}))+\varphi_{D}^{q}\left(F\left(\hat{x}, x_{0}\right)\right) .
$$

Therefore, by (3.12) we have

$$
\varphi_{D}^{q}\left(F\left(\bar{x}, x_{0}\right)\right)+d(\bar{x}, \hat{x}) \leq \varphi_{D}^{q}(F(\bar{x}, \hat{x}))+\varphi_{D}^{q}\left(F\left(\hat{x}, x_{0}\right)\right)+d(\bar{x}, \hat{x}) \leq \varphi_{D}^{q}\left(F\left(\hat{x}, x_{0}\right)\right),
$$

which is contrary to (3.11). Thus, part (3) is proved.

Parts (4)-(6) follow by applying assertions (1)-(3) to the distance $d^{\prime}: X \times X \rightarrow \mathbb{R}, d^{\prime}\left(x_{1}, x_{2}\right):=$ $\frac{\varepsilon \delta_{q}^{C}}{\lambda} d\left(x_{1}, x_{2}\right)$, for all $x_{1}, x_{2} \in X$.

Remark 3.5. Theorem 3.6 encompasses and improves [15, Theorem 3], which requires stronger lower semicontinuity and triangle inequality assumptions (see Remarks 3.2 and 3.4 and [15, Lemma 4]). 
From Lemma 3.4 we obtain the following formulation of Theorem 3.6 that works provided that the bifunction $F: X \times X \rightarrow Y$ is diagonal null, i.e., whenever $F(x, x)=0$, for all $x \in X$.

Theorem 3.7. Consider problem (VEP) with $S=X, C \in \mathscr{C}, \varepsilon>0, x_{0} \in W \operatorname{Sol}(F, X, C, \varepsilon)$ and $q \in \operatorname{int} D$. Suppose that $F\left(\cdot, x_{0}\right): X \rightarrow Y$ is strict $q$-decreasingly D-lower-semicontinuous, $F$ is diagonal null and satisfies the triangle inequality property in $-D$ by $q$ at $x_{0}$. Then, there exists $\hat{x} \in X$ such that

(1) $F\left(\hat{x}, x_{0}\right)+d\left(\hat{x}, x_{0}\right) q \leq_{D} F\left(x_{0}, x_{0}\right)$.

(2) $d\left(\hat{x}, x_{0}\right) \leq \varepsilon \delta_{q}^{C}$.

(3) $F(x, \hat{x})+d(x, \hat{x}) q \not_{D} F(\hat{x}, \hat{x})$, for all $x \in X \backslash\{\hat{x}\}$.

If in addition $C \in \mathscr{C}_{q}$, then, for each $\lambda>0$, there exists $\hat{x}_{\lambda} \in X$ such that

(4) $F\left(\hat{x}_{\lambda}, x_{0}\right)+\frac{\varepsilon \delta_{q}^{C}}{\lambda} d\left(\hat{x}_{\lambda}, x_{0}\right) q \leq_{D} F\left(x_{0}, x_{0}\right)$.

(5) $d\left(\hat{x}_{\lambda}, x_{0}\right) \leq \lambda$.

(6) $F(x, \hat{x})+\frac{\varepsilon \delta_{q}^{C}}{\lambda} d\left(x, \hat{x}_{\lambda}\right) q \not \underline{X}_{D} F\left(\hat{x}_{\lambda}, \hat{x}_{\lambda}\right)$, for all $x \in X \backslash\left\{\hat{x}_{\lambda}\right\}$.

\section{Acknowledgments}

The author is grateful to Professor Lidia Huerga for her helpful comments and suggestions. This work was supported by Ministerio de Ciencia, Innovación y Universidades (MCIU), Agencia Estatal de Investigación (AEI) (Spain) and Fondo Europeo de Desarrollo Regional (FEDER) under project PGC2018-096899-B-I00 (MCIU/AEI/FEDER, UE).

\section{REFERENCES}

[1] Q.H. Ansari, Vectorial form of Ekeland-type variational principle with applications to vector equilibrium problems and fixed point theory, J. Math. Anal. Appl. 334 (2007), 561-575.

[2] Q.H. Ansari, E. Köbis, J.-C. Yao, Vector Variational Inequalities and Vector Optimization. Theory and Applications, Springer, Cham, 2018.

[3] Q.H. Ansari, J.-C. Yao (eds.), Recent Developments in Vector Optimization, Springer-Verlag, Berlin, 2012.

[4] Y. Araya, K. Kimura, T. Tanaka, Existence of vector equilibria via Ekeland's variational principle, Taiwanese J. Math. 12 (2008), 1991-2000.

[5] T.Q. Bao, S. Cobzaş, A. Soubeyran, Variational principles, completeness and the existence of traps in behavioral sciences, Ann. Oper. Res. 269 (2018), 53-79.

[6] M. Bianchi, G. Kassay, R. Pini, Ekeland's principle for vector equilibrium problems, Nonlinear Anal. 66 (2007), 1454-1464.

[7] G.Y. Chen, X.X. Huang, X.Q. Yang, Vector Optimization. Set-Valued and Variational Analysis, Springer, Berlin, 2005.

[8] I. Ekeland, On the variational principle, J. Math. Anal. Appl. 47 (1974), 324-353.

[9] I. Ekeland, Nonconvex minimization problems, Bull. Amer. Math. Soc. (N.S.) 1 (1979), 443-474.

[10] C. Finet, L. Quarta, Vector-valued perturbed equilibrium problems, J. Math. Anal. Appl. 343 (2008), 531-545.

[11] F. Giannessi (ed.), Vector Variational Inequalities and Vector Equilibria. Mathematical Theories, Kluwer Academic Publishers, Dordrecht, 2000.

[12] A. Göpfert, H. Rihai, C. Tammer, and C. Zălinescu, Variational Methods in Partially Ordered Spaces, Springer-Verlag, New York, 2003.

[13] C. Gutiérrez, L. Huerga, B. Jiménez, V. Novo, Henig approximate proper efficiency and optimization problems with difference of vector mappings, J. Convex Anal. 23 (2016), 661-690.

[14] C. Gutiérrez, G. Kassay, V. Novo, J.L. Ródenas-Pedregosa, Ekeland variational principles in vector equilibrium problems, SIAM J. Optim. 27 (2017), 2405-2425. 
[15] L.P. Hai, L. Huerga, P.Q. Khanh, V. Novo, Variants of the Ekeland variational principle for approximate proper solutions of vector equilibrium problems, J. Global Optim. 74 (2019), 361-382.

[16] E. Hernández, L. Rodríguez-Marín, Nonconvex scalarization in set optimization with set-valued maps, J. Math. Anal. Appl. 325 (2007), 1-18.

[17] G. Kassay, V. Rădulescu, Equilibrium Problems and Applications, Academic Press, London, 2019.

[18] A.A. Khan, C. Tammer, C. Zălinescu, Set-valued Optimization. An Introduction with Applications, SpringerVerlag, Berlin, 2015.

[19] M. Miholca, Cyclically antimonotone vector equilibrium problems, Optimization 67 (2018), 2191-2204.

[20] J.H. Qiu, An equilibrium version of vectorial Ekeland variational principle and its applications to equilibrium problems, Nonlinear Anal. Real World Appl. 27 (2016), 26-42.

[21] J.H. Qiu, Vectorial Ekeland variational principle and cyclically antimonotone equilibrium problems, Acta Math. Sci. Ser. B (Engl. Ed.) 39B(2) (2019), 1-22.

[22] J.H. Qiu, F. He, Ekeland variational principles for set-valued functions with set perturbations, Optimization (2019), DOI: 10.1080/02331934.2019.1653295

[23] C. Tammer, A generalization of Ekeland's variational principle, Optimization 25 (1992), 129-141. 